\title{
Recent NASA Research on Aerodynamic Modeling of Post- Stall and Spin Dynamics of Large Transport Airplanes
}

\author{
Austin M. Murch ${ }^{1}$ and John V. Foster ${ }^{2}$ \\ NASA Langley Research Center, Hampton, VA, 23681-2199
}

\begin{abstract}
A simulation study was conducted to investigate aerodynamic modeling methods for prediction of post-stall flight dynamics of large transport airplanes. The research approach involved integrating dynamic wind tunnel data from rotary balance and forced oscillation testing with static wind tunnel data to predict aerodynamic forces and moments during highly dynamic departure and spin motions. Several state-of-the-art aerodynamic modeling methods were evaluated and predicted flight dynamics using these various approaches were compared. Results showed the different modeling methods had varying effects on the predicted flight dynamics and the differences were most significant during uncoordinated maneuvers. Preliminary wind tunnel validation data indicated the potential of the various methods for predicting steady spin motions.
\end{abstract}

\section{Nomenclature}

$\alpha \quad=$ angle of attack, degrees

$\alpha_{\text {ave }} \quad=$ average value of $\alpha$

$\beta \quad=$ angle of sideslip, degrees

$\beta_{\text {ave }} \quad=$ average value of $\beta$

$p_{b} \quad=$ body-axis roll rate, degrees/second or radians/second

$q_{b} \quad=$ body-axis pitch rate, degrees/second or radians/second

$r_{b} \quad=$ body-axis yaw rate, degrees/second or radians/second

$\bar{\Omega} \quad=$ total angular rate, degrees/second

$\bar{\Omega}_{x z} \quad=$ projection of the total angular rate into the body-axis x-z plane, degrees/second

$\omega=$ = wind-axis (velocity-vector) roll rate, degrees/second or radians/second

$\omega_{\text {ave }} \quad=$ average value of $\omega$

$b \quad=$ wingspan, feet

$L \quad=$ length, feet

$\bar{c} \quad=$ mean aerodynamic chord, feet

$V \quad=$ airspeed, feet/second

$\hat{p} \quad=$ nondimensional body-axis roll rate, $\frac{p_{b} b}{2 V}$

$\hat{q} \quad=$ nondimensional body -axis pitch rate, $\frac{q_{b} \bar{c}}{2 V}$

$\hat{r} \quad=\quad$ nondimensional body -axis yaw rate, $\frac{r_{b} b}{2 V}$

$\hat{\omega}=$ nondimensional wind-axis roll rate, $\frac{\omega b}{2 V}$

$p_{\text {osc }}=$ oscillatory component assigned to body-axis roll rate, degrees/second

\footnotetext{
${ }^{1}$ Research Engineer, Flight Dynamics Branch, Mail Stop 308, AIAA Member.

${ }^{2}$ Senior Research Engineer, Flight Dynamics Branch, Mail Stop 308, AIAA Associate Fellow.
} 


$\begin{array}{ll}q_{o s c} & =\text { oscillatory component assigned to body-axis pitch rate, degrees/second } \\ r_{\text {osc }} & =\text { oscillatory component assigned to body-axis yaw rate, degrees/second } \\ \bar{\Omega}_{\text {osc }} & =\text { total oscillatory angular rate component, degrees/second } \\ \omega_{s s} & =\text { steady-state component assigned to wind-axis roll rate, degrees/second } \\ C_{l} & =\text { aerodynamic rolling moment coefficient } \\ C_{m} & =\text { aerodynamic pitching moment coefficient } \\ C_{n} & =\text { aerodynamic yawing moment coefficient } \\ \Delta & =\text { denotes increment } \\ \delta_{r} & =\text { rudder deflection, positive trailing edge left, degrees } \\ \delta_{e} & =\text { elevator deflection, positive trailing edge down, degrees } \\ \delta_{a_{R}} & =\text { right aileron deflection, positive trailing edge down, degrees } \\ \delta_{a_{L}} & =\text { left aileron deflection, positive trailing edge down, degrees } \\ C G & =\text { center of gravity, percent mean aerodynamic chord } \\ D I R & =\text { direct resolution blending method } \\ 2 D-K A L & =2 \mathrm{D} \text { Kalviste blending method } \\ H Y-K A L & =\text { Hybrid Kalviste blending method } \\ E X R R & =\text { Excess Roll Rate blending method } \\ F O & =\text { forced oscillation } \\ R B & =\text { rotary balance } \\ N A S A & =\text { National Aeronautics and Space Administration } \\ L a R C & =\text { Langley Research Center }\end{array}$

\section{Introduction}

S part of the NASA Aviation Safety Program, research has been conducted to develop aerodynamic modeling A methods for simulations that accurately predict the flight dynamics characteristics of large transport airplanes in upset conditions ${ }^{1,2}$. The motivation for this research stems from the recognition that simulation is a vital tool for addressing loss-of-control accidents, including applications to pilot training, accident reconstruction, and advanced control system analysis. The ultimate goal of this effort is to contribute to the reduction of the fatality rate due to loss-of-control accidents.

An important part of aerodynamic modeling for loss-of-control scenarios involves prediction of stall, departure and incipient spin motions that have been observed during some accidents. Significant advances in aerodynamic modeling of fighter configurations in post-stall and spin conditions have been achieved over the past several decades, but it is only recently that simulations of transport configurations in these flight regimes has gained interest. However, it has been recognized that flight validation of full-scale transport airplanes in post-stall regimes, in contrast to fighter configurations, is severely limited due to the unacceptable flight safety risks. As a result, the use of unmanned subscale flight vehicles has been proposed as an alternative for validation of simulations in high-risk conditions ${ }^{3}$.

Current research is aimed at assessing and advancing state-of-the-art modeling methods for transport configurations and studying key flight dynamics characteristics of loss-of-control events. The research approach involves integrating dynamic wind tunnel data from rotary balance and forced oscillation testing with static wind tunnel data to predict rigid-body aerodynamic forces and moments during highly dynamic post-stall/departure motions. Previous research has been primarily aimed at methods to merge and blend data from dynamic test methods in a way that emulates the aircraft motions ${ }^{4,5,6}$. Specifically, measurements from oscillatory motions (see Fig. 1) are combined with those from steady-state rotary motions (see Fig. 2) with the goal of predicting a range of motions typical of loss-of-control events. Results from previous research have indicated the need for advanced wind tunnel test rigs (e.g. combined motion rigs) that more closely replicate real aircraft motions and have highlighted the need for data reduction methods that better characterize and identify highly non-linear and time-dependent aerodynamic properties that are typical of separated flows.

This paper shows results of applying several state-of-the-art aerodynamic modeling methods using dynamic wind tunnel data, for the purpose of predicting post-stall flight motions, along with comparisons of predicted flight dynamics using these various approaches. Results from a preliminary free-spin test of a subscale transport model (see Fig. 3) will be compared to simulation results as an initial validation of the modeling methods. Finally, 
recommendations regarding wind tunnel test methods, aerodynamic modeling approaches, and validation requirements will be discussed.

\section{Approach}

The approach to this research was to apply state-of-the-art aerodynamic modeling methods for post-stall and spin motions to a transport configuration. The objective was to identify advantages, disadvantages, and/or limitations of these methods and to recommend future research needed to improve prediction of post-stall and spin flight dynamics.

\section{A. Background}

The approach to aerodynamic modeling typically involves summation of static effects (e.g. angle of attack, control position) with dynamic effects (e.g. angular rate), the latter often referred to as rate damping effects. The typical practice used today for large transport simulators is that static effects are normally based on wind tunnel measurements but prediction of the dynamic effects are usually accomplished using empirical methods ${ }^{7}$ or from flight test data using small-amplitude dynamic maneuvers. These simulation models of large transport flight behavior are normally not designed for upset conditions and therefore are limited to the normal flight envelope below stall angles of attack. Recent analysis of loss-of-control accidents ${ }^{8}$ revealed that the normal flight envelope was often exceeded during loss-of-control accidents, and angles of attack well beyond stall were observed. Further simulation studies ${ }^{7}$ have also shown that accurate modeling of angular rate effects on large transports had a significant effect on the predicted aircraft behavior, which exposed the need for more detailed investigation of the post-stall dynamic behavior.

Aerodynamic modeling of highly dynamic maneuvers such as post-stall gyrations, spins, and other out-of-control motions is not a new problem. In the past thirty years, these motions have been modeled for numerous fighter aircraft configurations $5,6,9,10,11$. In addition, stall/spin accidents led to modeling research of general aviation aircraft in the 1980's, including studies by NASA ${ }^{12}$. In both cases, modeling the angular rate effects relied on two existing experimental test methods; oscillatory motion rigs (i.e. forced oscillation) and steady motion rigs (i.e. rotary balance). However, out-of-control motions often involve a combination of large amplitude, uncoordinated (i.e., the angular rate vector is not closely aligned with the velocity vector), and coupled motions which are difficult to replicate with existing wind tunnel motion rigs. As a result, previous research focused on methods to blend oscillatory and steady rate effects and on the development of new motion rigs designed to more closely simulate real flight motions.

One approach to modeling dynamic effects is to combine the forced oscillation and rotary balance data sets based on the characteristics of aircraft motion. The underlying assumption in this approach is that data from two fundamentally different dynamic wind tunnel test motions can be combined vectorially to approximate arbitrary dynamic motion of aircraft. While this approach presents many challenges in terms of modeling and similitude requirements, it has been used successfully and is the most commonly used approach to date. Several different blending methods using this vectorial technique have been used effectively on fighter aircraft in the past. However, application of these methods to large transport configurations has been limited and is a motivating factor for this research.

Although experience with out-of-control flight motions of transport airplanes is very limited, recent research has shown significant differences in dynamic wind tunnel test results between fighter and transport configurations ${ }^{13}$. As shown in Table I, comparison of flight data from loss-of-control motions of fighter aircraft ${ }^{14}$ and commercial transports ${ }^{7}$ indicate both configurations can reach high post-stall wind incidence angles and similar nondimensional rates during loss-of-control motions. However, in general, transport configurations are not expected to achieve as high wind incidence angles as fighters. A goal of this research is to identify the applicability of current blending methods to transport configurations and the following section provides a description of the blending methods used.

Table I. Comparison of Fighter and Commercial Aircraft Loss-of-Control Motions

\begin{tabular}{|c|c|c|c|c|c|}
\hline Configuration & $\alpha_{\max }$ & $\beta_{\max }$ & $\hat{p}_{\max }$ & $\hat{q}_{\max }$ & $\hat{r}_{\max }$ \\
\hline Fighter $^{14}$ & $70^{\circ}$ & $45^{\circ}$ & 0.129 & 0.0062 & 0.043 \\
\hline Transport $^{7}$ & $47^{\circ}$ & $20^{\circ}$ & 0.126 & 0.0045 & 0.047 \\
\hline
\end{tabular}




\section{B. Description of Blending Methods}

The most common blending approach is to partition the total angular rate vector $(\bar{\Omega})$ into a "steady-state" component $\left(\omega_{s s}\right)$ along the velocity vector (corresponding to rotary balance motion) and "oscillatory" components $\left(\bar{\Omega}_{\text {osc }}\right)$ along the aircraft body axes (corresponding to forced oscillation motion). As illustrated in Fig. 4, these components are then used with the forced oscillation and rotary balance databases to determine the total dynamic contribution to the aerodynamic forces and moments. The specific assignments of the steady and oscillatory rate components vary from method to method, and limitations have been noted for each. The most commonly used blending methods are the Direct Resolution method, several variants of the Kalviste method, the Excess Roll Rate method, and using forced oscillation data only. A brief description of each method used in this research is as follows:

\section{Direct Resolution Method}

Direct resolution is perhaps the simplest blending method ${ }^{15}$. The total angular rate vector is decomposed into the wind-axis roll rate (the "steady-state" component) and a residual component orthogonal to the velocity vector, which is further resolved into body-axis "oscillatory" components.

$$
\begin{aligned}
\omega_{s s} & =p_{b} \cos \alpha \cos \beta+q_{b} \sin \beta+r_{b} \sin \alpha \cos \beta \\
p_{\text {osc }} & =p_{b}-\omega_{s s} \cos \alpha \cos \beta \\
q_{\text {osc }} & =q_{b}-\omega_{s s} \sin \beta \\
r_{\text {osc }} & =r_{b}-\omega_{s s} \sin \alpha \cos \beta
\end{aligned}
$$

One issue with this method is the oscillatory roll and yaw components often have opposite signs of the corresponding body-axis roll and yaw rates. For highly uncoordinated maneuvers at higher angles of attack, this anomaly can be a significant problem as the forced oscillation data can become highly nonlinear and/or asymmetric with respect to angular rate.

\section{Kalviste Methods}

Since Kalviste's original formulation ${ }^{4}$ (often called 3D Kalviste), at least two additional variants have been used; 2D Kalviste and Hybrid Kalviste. The latter two methods have seen the most use in recent years due to shortcomings of the original 3D Kalviste method, and were the only Kalviste methods used in this research. The 2D Kalviste and Hybrid Kalviste methods are similar, using the position of the angular rate vector relative to the velocity vector to determine the steady-state and oscillatory components. There are three possible cases, as illustrated in Fig. 5. In case 1, the projection of the total angular rate vector into the x-z plane $\left(\bar{\Omega}_{x z}\right)$ is closer to the body yaw-axis, and is decomposed into a wind-axis component and a body-axis yaw component; the body-axis roll component is not used. In case 2, the total angular rate vector is closer to the body roll-axis, and is decomposed into a wind-axis component and a body-axis roll component; the body-axis yaw component is not used. In case 3, the angular rate is highly uncoordinated (roll and yaw have opposite signs), no wind-axis data is used and the method uses body-axis components only. The 2D and Hybrid methods differ only in the equations used to compute the various components - the 2D Kalviste method does not account for sideslip angle, while Hybrid Kalviste includes the effect of sideslip. The Hybrid Kalviste equations are given in Table II. The 2D Kalviste equations can be obtained by setting the sideslip angle equal to zero in the Hybrid Kalviste equations.

Table II. Equations used in Hybrid Kalviste method

\begin{tabular}{|c|c|c|c|}
\hline Angular Rate Term & Case 1 & Case 2 & Case 3 \\
\hline$\omega_{s s}=$ & $\frac{p_{b}}{\cos \alpha \cos \beta}$ & $\frac{r_{b}}{\sin \alpha \cos \beta}$ & 0 \\
\hline$p_{o s c}=$ & 0 & $p_{b}-\omega_{s s} \cos \alpha \cos \beta$ & $p_{b}$ \\
\hline$q_{o s c}=$ & $q_{b}-\omega_{s s} \sin \beta$ & $q_{b}-\omega_{s s} \sin \beta$ & $q_{b}$ \\
\hline$r_{o s c}=$ & $r_{b}-\omega_{s s} \sin \alpha \cos \beta$ & 0 & $r_{b}$ \\
\hline
\end{tabular}


Previous research efforts found the Kalviste methods to be sensitive to slightly uncoordinated maneuvers at very high angles of attack, which can cause the method to abruptly switch to using forced oscillation data only ${ }^{6}$. Due to the lower magnitude of wind angles experienced with transports, this sensitivity was not found in this research.

\section{Excess Roll Rate Method}

The Excess Roll Rate method ${ }^{6}$ does not use a body-axis yaw component, and it uses the same equations as Case 2 of the Hybrid Kalviste method. This approach was intended to model roll rate-dominated motions and avoids complexities associated with using yaw forced oscillation data. One significant drawback of this method is the presence of a singularity at $\alpha=0^{\circ}$, which necessitates a different approach at low angles of attack. For this research, Hybrid Kalviste was used if the angle of attack was less than $15^{\circ}$.

\section{Forced Oscillation Method}

Previous research on blending methods found that using forced oscillation data alone (i.e., Case 3 of the Hybrid Kalviste method) was not adequate to model highly dynamic post-stall motions ${ }^{6,10}$. For completeness, this method was studied and compared to the other blending methods. Results from the forced oscillation studies are not shown herein but are briefly discussed. One problem with this approach is that the angular rates attained in maneuvers such as spins can exceed the limits of the forced oscillation database, invalidating the simulations results and possibly causing the simulation to diverge.

\section{Research Methodology}

Two approaches were used to analyze the various blending methods. The first approach focused on the differences between the blending methods and examined how each method decomposed the angular rates for an oscillatory spin motion. The steady-state and oscillatory rate components from each method were compared, as well as the aerodynamic coefficient increments produced from the aerodynamic model. The second approach was to examine the effect of the blending method on post-stall and spin dynamics by implementing each method in a realtime MATLAB/Simulink-based simulation of a 5.5\% dynamically-scaled commercial transport model ${ }^{16}$ (see Fig. 6). The simulation utilizes an extensive static wind tunnel-derived database developed as part of previous aerodynamic modeling studies ${ }^{1}$. Databases from the aforementioned dynamic (forced oscillation and rotary balance) wind tunnel tests were included in the simulations. The rotary balance database modeled effects of the six degree-of-freedom aerodynamic coefficients and was created as nonlinear look-up tables that are functions of angle of attack, sideslip angle, and nondimensional angular rate. The forced oscillation database was created as nonlinear look up tables that are functions of angle of attack and nondimensional body-axis rate. Effects of nondimensional roll and yaw rate were modeled for the aerodynamic side force, rolling moment, and yawing moment coefficients. Effects of nondimensional pitch rate were modeled for the aerodynamic axial force, normal force, and pitching moment coefficients.

To analyze the effect of the blending methods on post-stall and spin dynamics, an extensive simulator test using numerous spin entry techniques was conducted. Using identical initial conditions and control inputs with each blending method, comparison of simulator time histories and key characteristics of the resulting motions provided insight into the effect of each blending method on the overall aircraft motion. Although the potential for a commercial transport to enter a fully developed spin is small, analysis of the incipient and fully-developed spin provided a good starting point because of the number of analysis tools available and extensive prior research.

Definitive conclusions as to which blending method, if any, provides the most accurate prediction of post-stall and spin dynamics require validation data. Validation of the modeling and blending methods is a difficult problem, given the unacceptable safety risks involved with operating commercial transports in the post-stall regime. For the purposes of this research, preliminary free-spin test results of a subscale model were used as an initial assessment of the blending methods.

\section{Results and Analysis}

\section{A. Blending Method Comparison}

The first task for this research focused on the differences between the blending methods and examined how each method decomposed an oscillatory spin motion. Figure 7 depicts a sample time history of angle of attack, sideslip angle, and body axis angular rates for an upright, moderately steep, oscillatory, right-hand spin that would be expected for a $5.5 \%$ scale transport aircraft based on simulation results. Figure 8 illustrates how each blending 
method would compute the angular rates with the steady state component $\left(\omega_{s s}\right)$ apportioned to the rotary balance data, and the oscillatory components $\left(p_{\text {osc }}, q_{\text {osc }}, r_{\text {osc }}\right.$ ) apportioned to the forced oscillation data (for clarity, only a one second time interval of the oscillation is shown). The blending methods agree in certain areas and differ widely in others. The areas of agreement in Fig. 8 can be correlated to the condition when the angular rate vector is closely aligned with the velocity vector, i.e., when the motion is well-coordinated. For well-coordinated motion, all of the blending methods yield approximately the same result, assigning most of the angular rate to the steady-state component. The areas of difference in Fig 8 can be correlated to the condition when the angular rate vector is not closely aligned with the velocity vector (i.e., the motion is uncoordinated). For uncoordinated motion, the blending methods can significantly differ in how the total angular rate vector is assigned to the steady and oscillatory rate components. For the oscillatory spin studied here, the angle between the velocity vector and the angular rate vector varies from $2^{\circ}$ to $31^{\circ}$.

The Direct Resolution method produces oscillatory roll and yaw rates that have signs opposite those of the body axis rates. In contrast, the Kalviste methods ensure the signs of the oscillatory roll and yaw components match the signs of the total body-axis rates. This can cause the steady state component to change rapidly, as can be seen in Fig. 8 near Time $=11.2$ seconds. The $2 \mathrm{D}$ and Hybrid Kalviste methods primarily differ in the oscillatory pitch component, which can have a pronounced effect on the pitching moment. The Excess Roll Rate method produces a relatively smooth decomposition with the largest steady state and oscillatory roll components. The oscillatory roll component also differs in sign with respect to the body axis values. The difference in sign between the oscillatory component and the body-axis rate could be a significant issue because there can be nonlinearities and asymmetries in the forced oscillation data.

While Fig. 8 illustrates how each blending method assigns the oscillatory and steady state components of the angular rate, it is important to examine how much effect each of the different components will have on the aerodynamic coefficients and therefore the overall predicted aircraft motion. Figures 9, 10, and 11 show the aerodynamic rolling, pitching, and yawing moments, respectively, as computed using each blending method. Each figure shows the contribution from the forced oscillation database (oscillatory component), rotary balance database (steady-state component), and the total moment coefficient (static and dynamic effects). In general, the Hybrid Kalviste and 2D Kalviste methods were nearly identical, with the exception of pitching moment. The Excess Roll Rate method often overlapped one or both of the Kalviste methods; this is not unexpected given the Excess Roll Rate method is the same as Case 2 of the Kalviste methods. The Direct Resolution method was most often different from the other methods.

For the rolling moment (Fig. 9), all of the methods generally agree, except that Direct Resolution and Excess Roll Rate have some areas of disagreement. Referring to Fig. 8, the differences for Excess Roll Rate stem from the steady state and roll oscillatory components, while the difference for Direct Resolution can be primarily attributed to the roll oscillatory component and the negative yaw oscillatory component. The effect of the negative yaw oscillatory component is more pronounced in the yaw axis, as can be seen in Fig. 11.

In the pitch axis (Fig. 10), all of the methods are in general agreement, with the exception of 2D Kalviste, and for some conditions, Excess Roll Rate. As shown in Fig. 8, these differences stem directly from the oscillatory pitch component, for which the 2D Kalviste and Excess Roll Rate methods produce the largest deviations from the other blending methods.

Figure 11 depicts the yawing moment, where the Kalviste methods overlap the entire time. The Direct Resolution method is in disagreement with the other methods primarily between Time $=11.2$ seconds and 11.6 seconds. The Excess Roll Rate method primarily disagrees with the other methods before Time $=11.2$ seconds and after 11.7 seconds. Referring again to Fig. 8, the difference with the Direct Resolution method is due to the yaw oscillatory component. The difference with the Excess Roll Rate method can be traced to the steady-state and oscillatory roll components, which are significantly greater in magnitude than what the other methods produce in those time intervals.

The approach of using forced oscillation data only was briefly included in this study. An important observation was that the magnitudes of the nondimensional angular rates attained in a modest spin often exceeded the boundaries of the forced oscillation database. This approach also produced significantly different moment coefficient predictions than the other blending methods and at times caused the simulation to diverge.

\section{B. Spin Simulation Analysis}

The second part of this research involved an extensive analysis of simulated spins using each blending method and a wide range of spin entries. In general, the different blending methods contributed to different incipient spin characteristics, but for most large pro-spin control inputs, a similar developed spin mode was reached. Only for certain partial pro-spin control inputs did the differences in incipient spin characteristics (caused in part by the 
blending method used) lead to a difference in the developed spin mode. This result can be explained by examining the situations where the blending methods have the greatest effect on the overall motion. Care must be taken when interpreting this result, since the blending methods, static and dynamic aerodynamic effects, and control effects are all a function of the wind incidence angles.

For well-coordinated maneuvers (e.g. nonoscillatory spins and velocity-vector rolls), the angular rate vector is closely aligned with the relative wind vector. This type of motion is well replicated by rotary balance motion and all of the blending methods studied will give nearly the same decomposition and attribute the majority of the angular rate to the steady state rate component. For this reason, very little difference can be discerned in simulation results of well-coordinated maneuvers using different blending methods. Figure 12 shows angle of attack plotted versus sideslip angle for an incipient and fully-developed nonoscillatory spin using each blending method. Note the difference in the incipient spin trajectories (which begin at $\alpha=23^{\circ}, \beta=0^{\circ}$ ) between blending methods; this is where the motion is uncoordinated. As the spin develops, the motion becomes more coordinated and each blending method gives similar results, so few differences can be discerned between the steady spins predicted by each method.

For uncoordinated maneuvers (e.g. incipient spins, oscillatory spins, and post-stall gyrations), the angular rate vector is not closely aligned with the relative wind vector. In this case each blending methods produces different dynamic aerodynamic contributions. The different dynamic effects produced by the blending methods are large enough to cause different incipient spin characteristics and different trajectories throughout the fully-developed spin phase, but typically not enough to affect the general characteristics of the spin (e.g. change an oscillatory spin into a nonoscillatory spin).

Figure 13 shows the angle of attack plotted versus sideslip angle for a fully-developed oscillatory spin using each blending method. The general characteristics of all of these spins are the same; upright, moderately steep, oscillatory, and high spin rates. Referring to the annotations on Fig. 13, note the average values for angle of attack, sideslip angle, and spin rate for all the spins are nearly the same. However the characteristics of the oscillations are very different when viewed in the $\alpha-\beta$ plane. The Direct Resolution method produces the least oscillatory spin, with the fastest spin rate and lowest average angle of attack. The 2D Kalviste method produced an oscillation of varying amplitude, while the Hybrid Kalviste and Excess Roll Rate methods had similar oscillation patterns. Note that both Kalviste methods and the Excess Roll Rate method had very similar average values of angle of attack, sideslip angle, and spin rate despite the differences in oscillation characteristics. While the blending methods exhibit the greatest dissimilarities during uncoordinated motion, the differences in trajectories noted here cannot be completely attributed to differences in the blending methods, since the static aerodynamics and control effects are also a function of the wind incidence angles. However, since the dynamic aerodynamic effects are significant for a developed spin, the dissimilarities in the blending methods will likely have a considerable effect on the oscillation characteristics.

In certain spin entries with partial pro-spin control inputs, the choice of blending method had a significant effect on the general characteristic of the spin. In these cases, the control effects are not as dominant in the overall aerodynamics, allowing the dynamic aerodynamic effects to play a more significant role in the incipient phase of the spin and which developed spin mode, if any, will be reached. For example, as depicted in Fig. 14, a spin entry with $\delta_{r}=17^{\circ} \delta_{e}=\delta_{a_{R}}=\delta_{a_{L}}=0^{\circ}$ did not produce a spin using the Direct Resolution, Hybrid Kalviste, or Excess Roll Rate methods, but produced an oscillatory spin using the 2D Kalviste method. This is one example of how dissimilarities in the dynamic aerodynamic effects produced by the blending methods can have a dramatic effect on the prediction of the developed motion.

In summary, this analysis indicated the choice of blending method will have the largest effect during uncoordinated maneuvers such as post-stall gyrations, incipient spins, and fully-developed oscillatory spins. Whether the impact of the blending method will be enough to radically affect the developed motion depends largely upon the control inputs used. This study showed that for less than full pro-spin control inputs, the dynamic aerodynamic effects can play a significant role in determining the developed motion, so that the choice of blending method becomes more important for prediction of post-stall and spin dynamics. Since loss-of-control motions rarely involve full, sustained pro-spin controls, the blending method used will likely be an important factor in accurately predicting these types of motion.

\section{Preliminary Free Spin Validation}

Limited preliminary results from free-spin testing of a $1 / 49^{\text {th }}$ scale transport model were used for comparison to the simulation results. These tests were conducted in the NASA LaRC 20-Foot Vertical Spin Tunnel, where a 
sustained, upright oscillatory spin was demonstrated using $\delta_{r}=30^{\circ} \quad \delta_{e}=\delta_{a_{R}}=\delta_{a_{L}}=0^{\circ}$ and $\mathrm{CG}=15.2 \% \bar{c}$. Simulations using the same scale, inertia loadings, control inputs, and similar initial conditions as the free-spin test also produced an upright oscillatory spin with similar nondimensional spin rate. Estimated nondimensional spin rate was the only data available from the free-spin test, and Fig. 15 shows this data compared to simulations using each blending method. While not a rigorous or complete validation, this preliminary result indicates that the blending methods evaluated give reasonable results for a transport aircraft configuration.

\section{Future Plans}

Future research will focus on validating the modeling methods. This activity will allow recommendations to be made regarding use of the four blending methods studied for modeling departures, incipient and fully-developed spin dynamics of transport aircraft. First, free-spin testing in the 20-Foot Vertical Spin Tunnel will be conducted to analyze a range of control inputs and spin conditions. Secondly, flight testing with unmanned subscale models is planned in order to validate post-stall and incipient spin phases. The validation activities may also lead to development of new modeling approaches and application to full-scale simulations.

\section{Conclusions}

Based upon the simulation study and limited free-spin test results, the following conclusions can be made:

1. Simulation results using each blending method exhibited different incipient spin characteristics, but for most large pro-spin control inputs a similar fully-developed spin mode was reached. Differences in the oscillation characteristics of oscillatory spin modes were noted for each blending method used. Only for certain partial prospin control inputs did the blending method lead to a difference in the developed spin mode obtained.

2. The largest differences between the blending methods occurred during uncoordinated maneuvers such as poststall gyrations, incipient spins, and oscillatory spins, and especially in cases with less than full control deflections. 3. The simulation study indicated that using forced oscillation data alone did not agree with any of the methods using rotary balance data. In addition, angular rates predicted by using forced oscillation data alone often exceeded the database limits.

4. All blending methods evaluated in this study predicted average spin rates that were in general agreement with preliminary free-spin test results.

5. Validation data is needed to determine the blending method that will produce the most accurate departure and spin predictions for transport aircraft configurations.

\section{References}

${ }^{1}$ Shah, G.H., Cunningham, K. Foster, J.V., Fremaux, C. M. Stewart, E.C., Wilborn, J. E., Gato, W., and Pratt, D. W., "WindTunnel Investigation of Commercial Transport Aircraft Aerodynamics at Extreme Flight Conditions," World Aviation Congress \& Display, SAE 2002-01-2912, Phoenix, AZ, November 2002.

${ }^{2}$ Cunningham, K., Foster, J. V., Shah, G. H., Stewart, E. C., Rivers, R. A., Wilborn, J. E., and Gato, W., "Simulation Study of a Commercial Transport Airplane During Stall and Post-Stall Flight," World Aviation Congress \& Dislplay, SAE 2004-01-3100, Reno, NV, November 2004.

${ }^{3}$ Jordan, T. L., Langford, W. M., Belcastro, Christine M., Foster, J. M., Shah, G. H., Howland, G., Kidd, R., "Development of a Dynamically Scaled Generic Transport Model Testbed for Flight Research Experiments", AUVSI Unmanned Systems North America 2004, AUVSI, Arlington, VA, 2004

${ }^{4}$ Kalviste, J., "Use of Rotary Balance and Forced Oscillation Test Data in a Six Degrees of Freedom Simulation," AIAA Atmospheric Flight Mechanics Conference, AIAA 82-1364, San Diego, CA, August 1982.

${ }^{5}$ Kramer, B. R., "Experimental Evaluation of Superposition Techniques Applied to Dynamic Aerodynamics (Invited)," $40^{\text {th }}$ AIAA Aerospace Sciences Meeting \& Exhibit, Reno, NV, January 2002.

${ }^{6}$ Kay, J.; "Acquiring and Modeling Unsteady Aerodynamic Characteristics," AIAA Atmospheric Flight Mechanics Conference, AIAA 200-3907, Denver, CO, August 2000.

${ }^{7}$ Wilborn, J. E., “An Analysis of Commercial Transport Aircraft Loss-of-Control Accidents and Intervention Strategies,” final report for NASA contract NAS1-20341, Task 10, June 29, 2001.

${ }^{8}$ Wilborn,, J. E., and Foster, J. V., “Defining Commercial Transport Loss-of-Control: A Quantitative Approach,” AIAA 
Atmospheric Flight Mechanics Conference, AIAA 2004-4811, Providence, RI, August 2004.

${ }^{9}$ Dickes, E. G., Ralston, J. N., and Lawson, K., “Application Of Large-Angle Data For Flight Simulation,” AIAA Modeling and Simulation Conference and Exhibit, AIAA 2000-4584, Denver, CO, August 2000.

${ }^{10}$ O'Connor, C. J., Ralston, J. N., and Fitzgerald, T., "Evaluation Of The NAWC/AD F/A-18 C/D Simulation Including Database Coverage And Dynamic Data Implementation Techniques," AIAA Atmospheric Flight Mechanics Conference, AIAA-1996-3365874, San Diego, CA, July 1996.

${ }^{11}$ Ogburn, M. E., Nguyen, L. T., and Hoffler, K. D., "Modeling of Large-Amplitude High-Angle-Of-Attack Maneuvers,” AIAA Atmospheric Flight Mechanics Conference, AIAA 88-4357, Minneapolis, MN, August 1988.

${ }^{12}$ Chambers, J. R., and Stough, H. P. III, "Summary of NASA Stall/Spin Research for General Aviation Configurations,” AIAA General Aviation Technology Conference, AIAA 86-2597, Anaheim, CA, 1986.

${ }^{13}$ Brandon, J. M., Foster, J. V., Shah, G. H., Gato, W., and Wilborn, J. E., "Comparison of Rolling Moment Characteristics During Roll Oscillations for a Low and a High Aspect Ratio Configuration," AIAA Atmospheric Flight Mechanics Conference, AIAA-2004-5273, Providence, RI, August 2004.

${ }^{14}$ Jamarillo, P. T., Ralston, J., "Simulation of the F/A-18D 'falling leaf'," AIAA Atmospheric Flight Mechanics Conference, AIAA 96-3371, San Diego, CA, July 1996.

${ }^{15}$ Birhle, W. Jr., and Barnhart, B., “Spin Prediction Techniques,” Journal of Aircraft, Vol. 20, No. 2, February 1983.

${ }^{16}$ Jordan, T. L., Langford, W. M., Hill, J. S., “Airborne Subscale Transport Aircraft Research Testbed: Aircraft Model Development," AIAA Guidance, Navigation, and Control Conference and Exhibit, AIAA 2005-6432, San Francisco, CA, August 2005. 
Figures

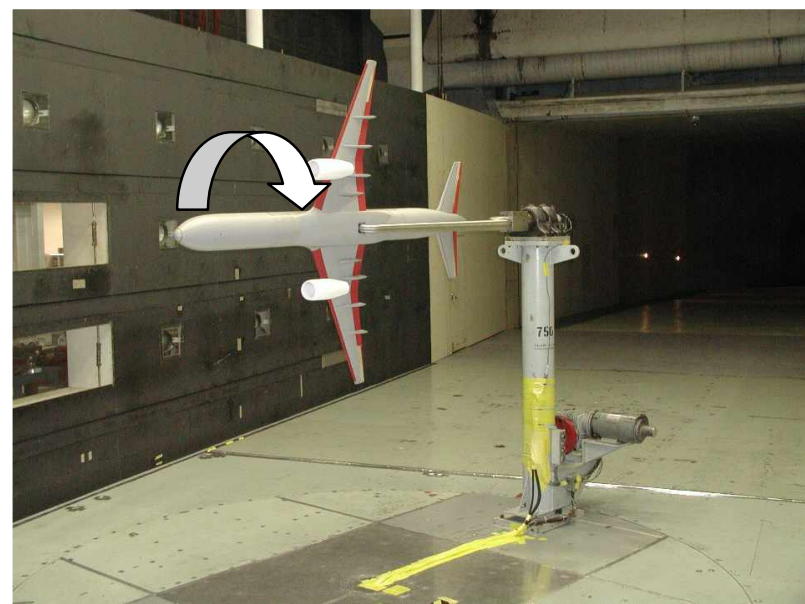

Figure 1. 5.5\% model on roll forced oscillation rig, NASA LaRC 14x22 Ft Tunnel.

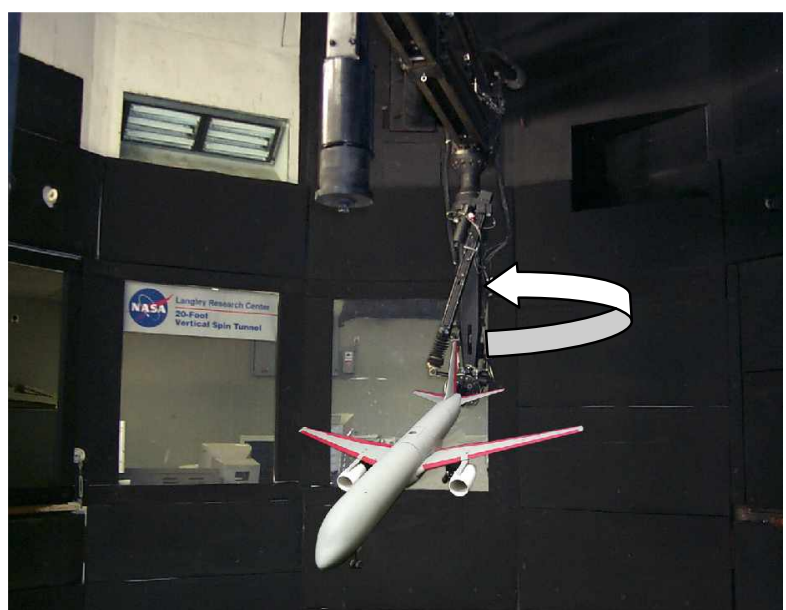

Figure 2. 3.5\% model on rotary balance rig, NASA LaRC 20 Ft Vertical Spin Tunnel.

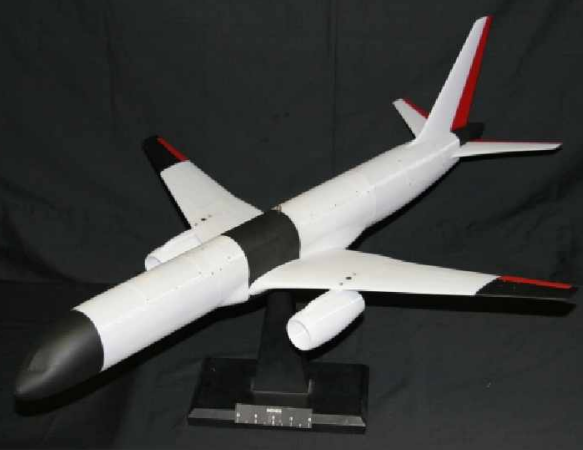

Figure 3. $1 / 49^{\text {th }}$ scale free-spin model. 


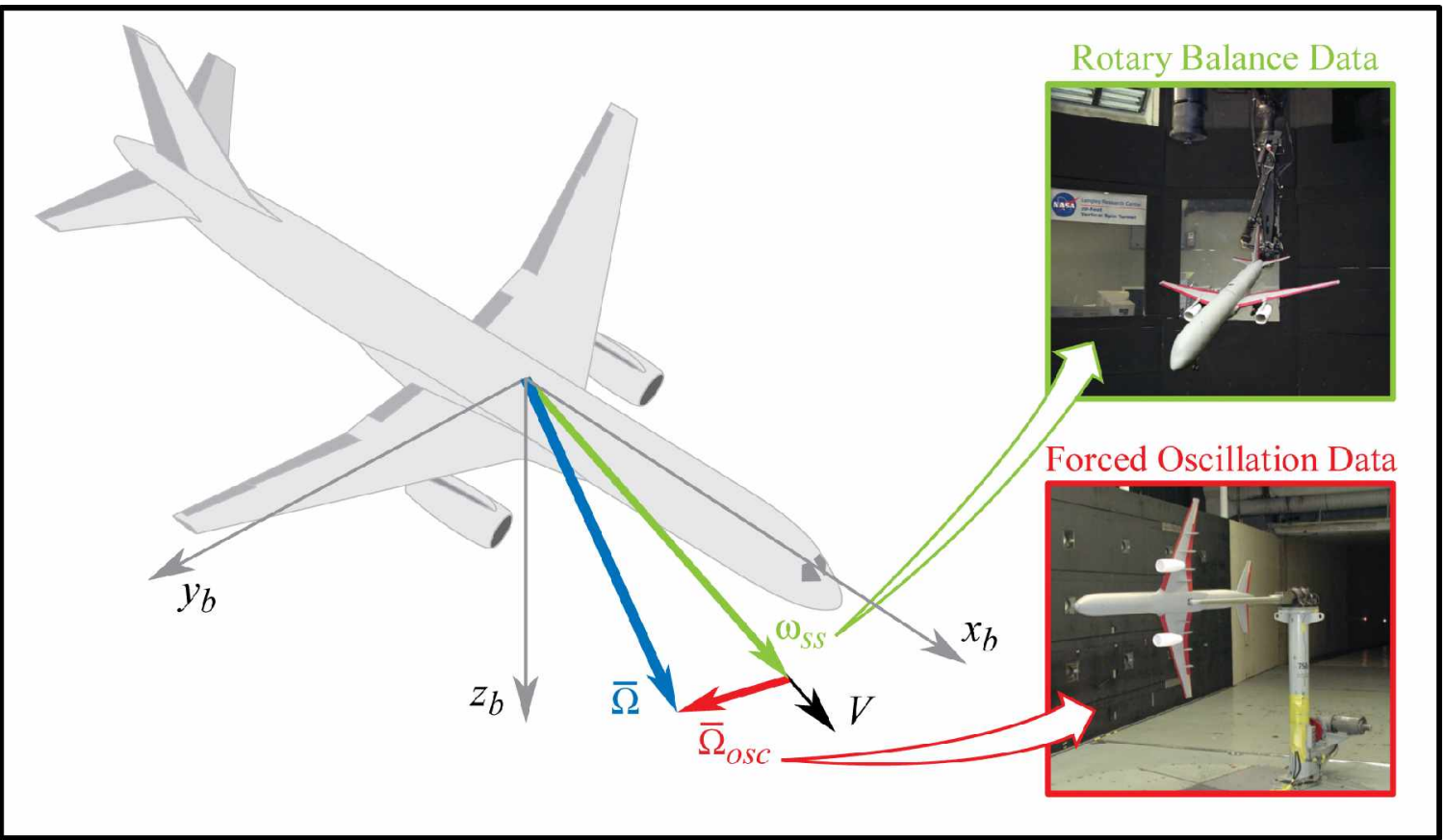

Figure 4. Decomposition of the total angular rate vector into steady-state and oscillatory components.
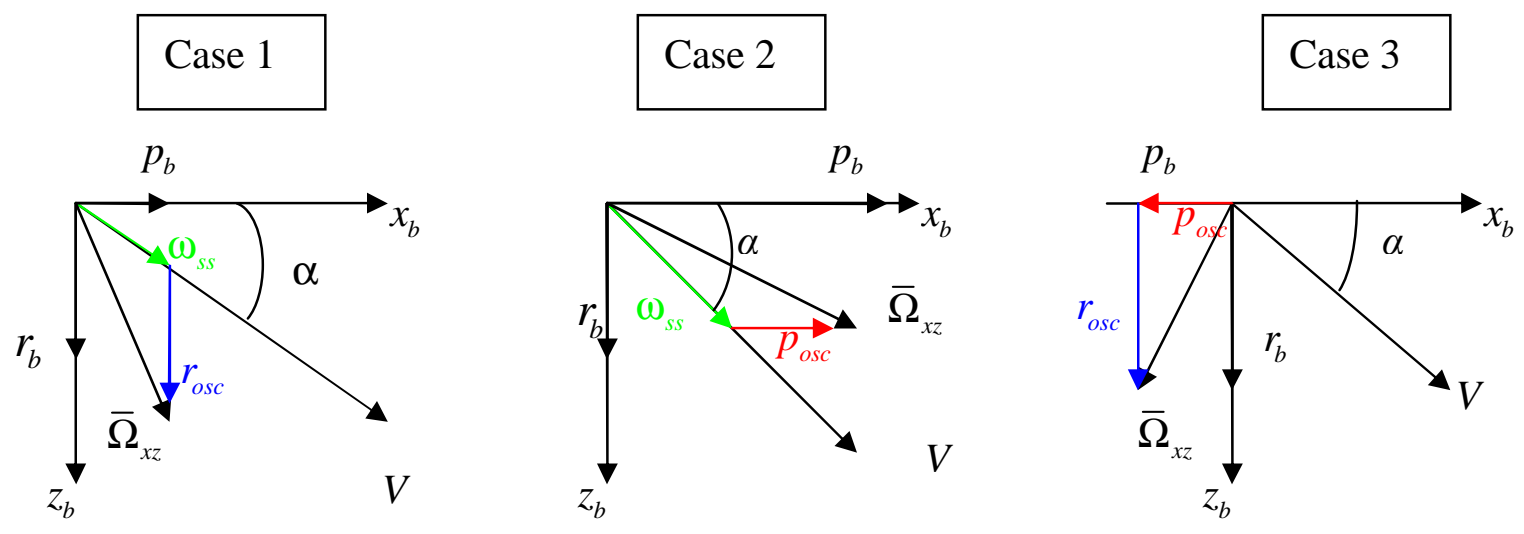

Figure 5. Three decomposition schemes used in the Kalviste methods. 


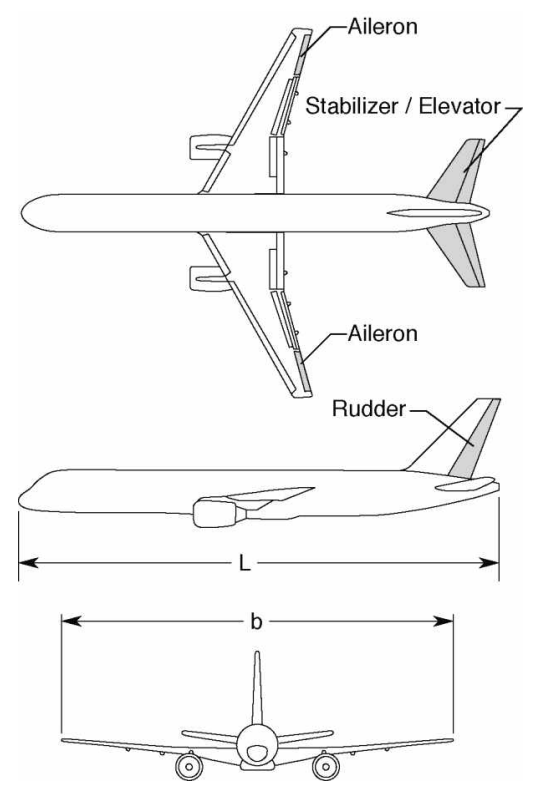

Figure 6. 3-view diagram of 5.5\% dynamically-scaled commercial transport model, $\mathrm{L}=8.54 \mathrm{ft}, \mathrm{b}=6.85 \mathrm{ft}, \mathrm{S}=5.90 \mathrm{ft}^{2}, \bar{c}=0.915 \mathrm{ft}$.
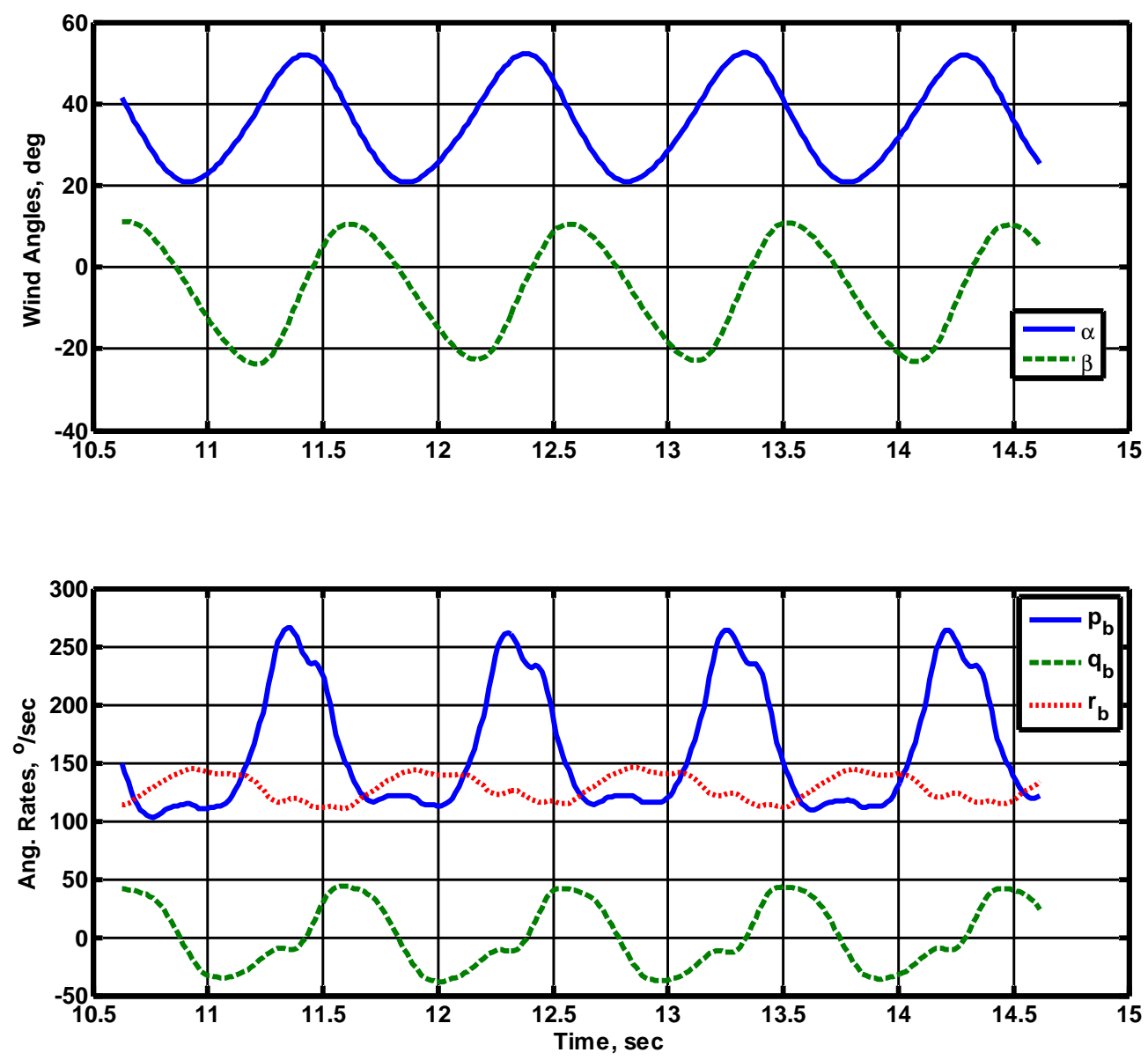

Figure 7. Sample simulation time history of body axis angular rates and wind angles for a fully-developed oscillatory spin, $5.5 \%$ scale. 

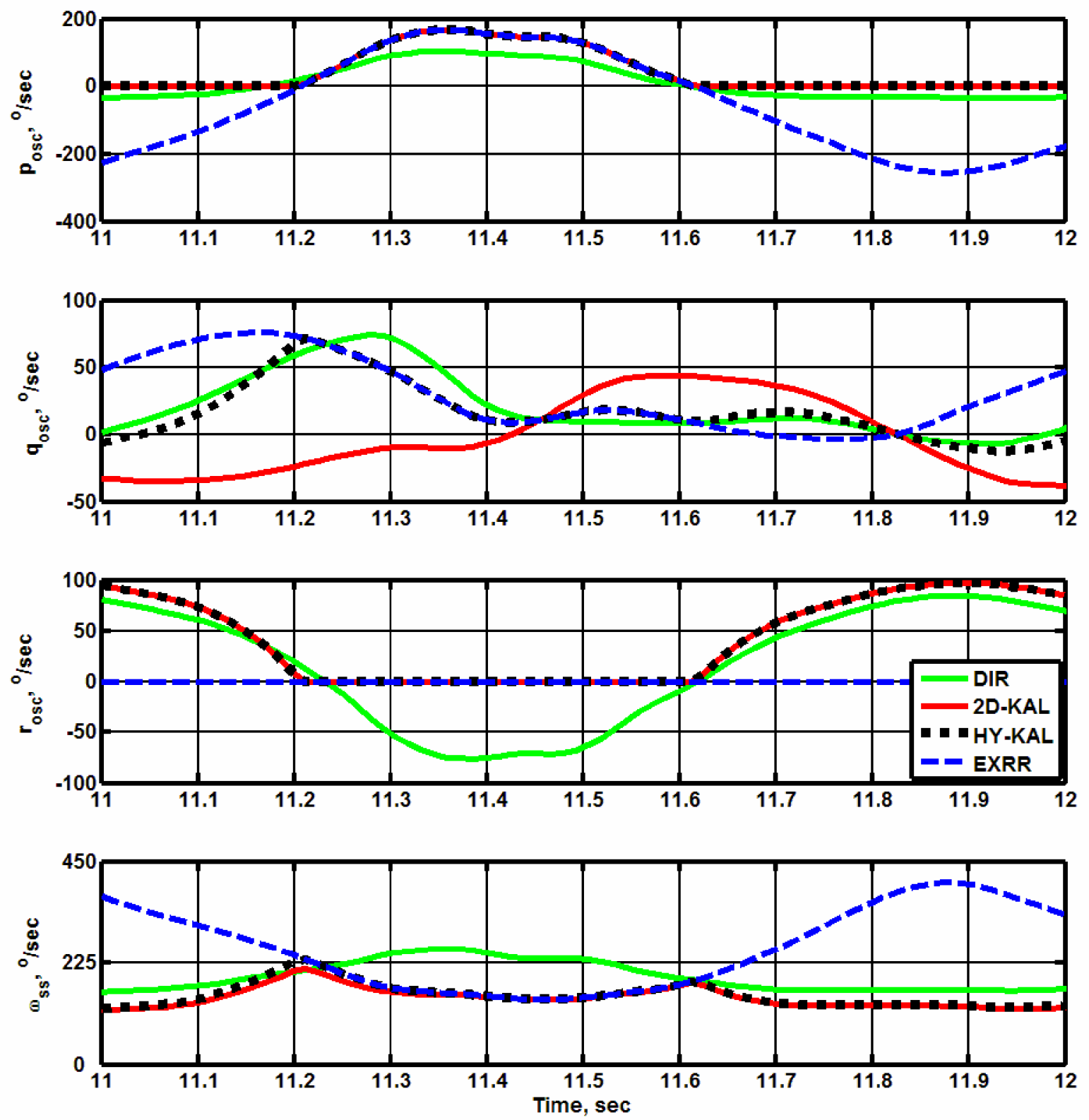

Figure 8. Oscillatory and steady state rate components for a one second time interval of a fully-developed oscillatory spin. 

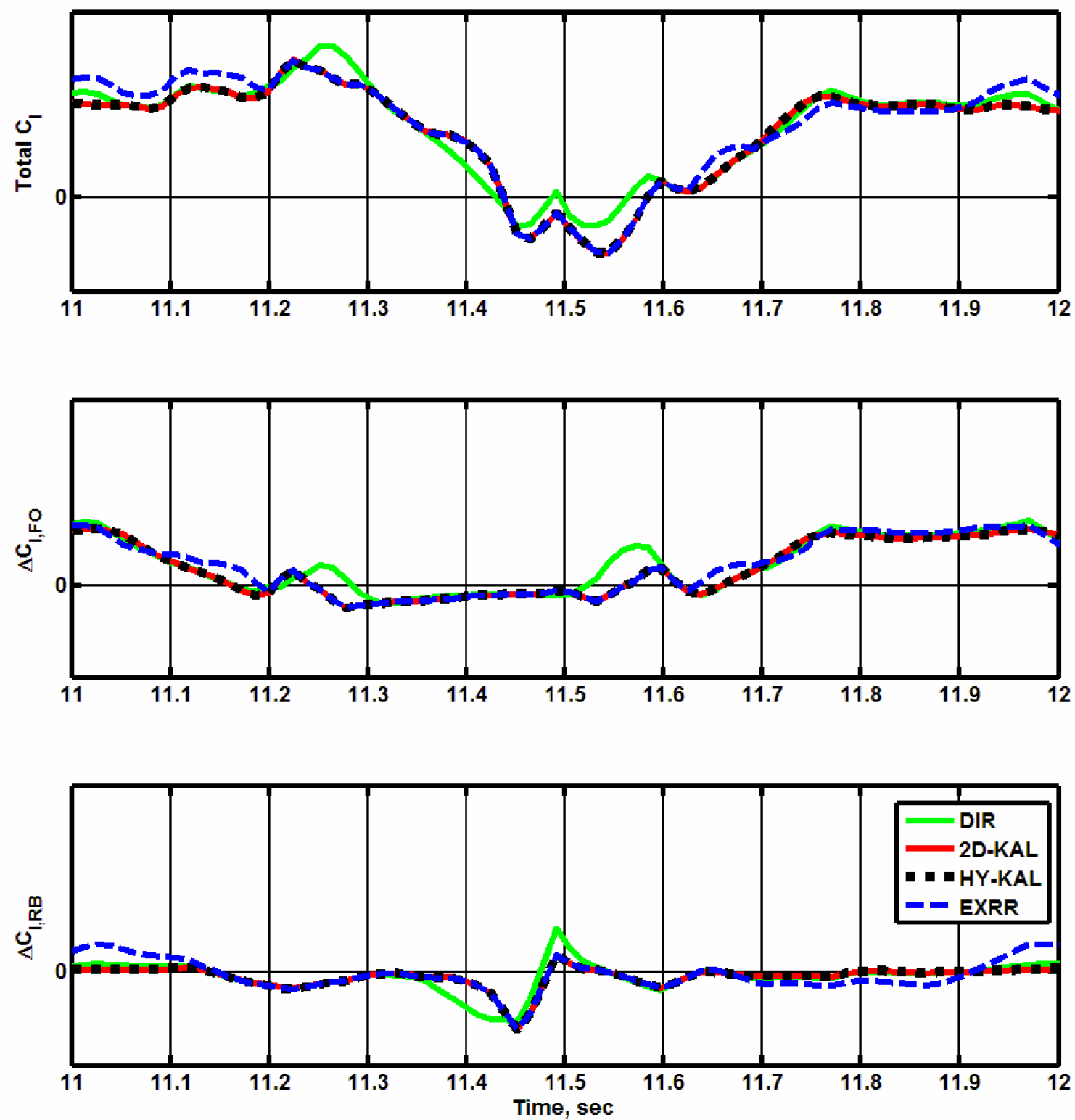

Figure 9. Rolling moment coefficients for a one second time interval of a fully-developed oscillatory spin: Total coefficient, forced oscillation increment, and rotary balance increment. 

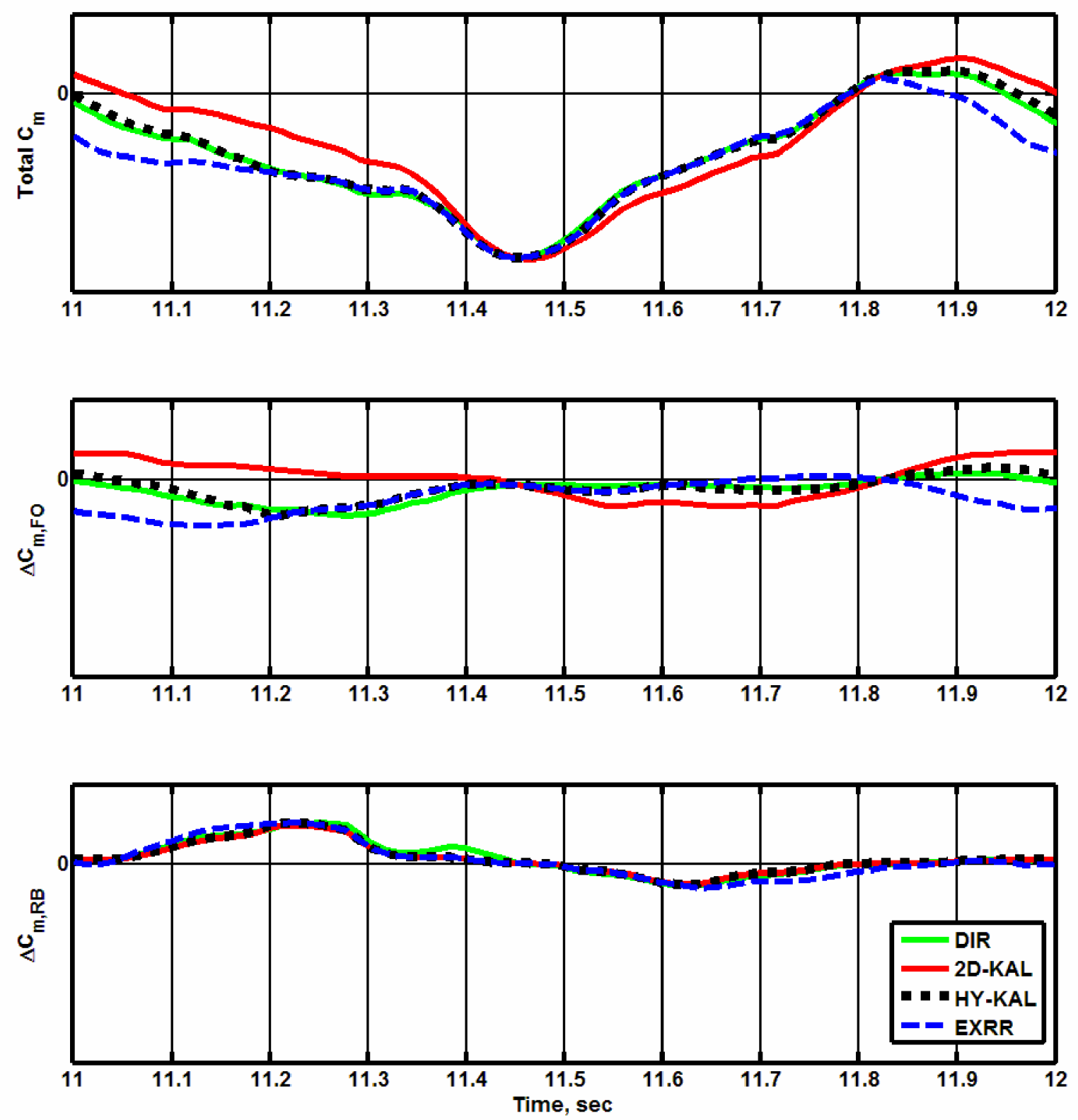

Figure 10. Pitching moment coefficients for a one second time interval of a fully-developed oscillatory spin: Total coefficient, forced oscillation increment, and rotary balance increment. 

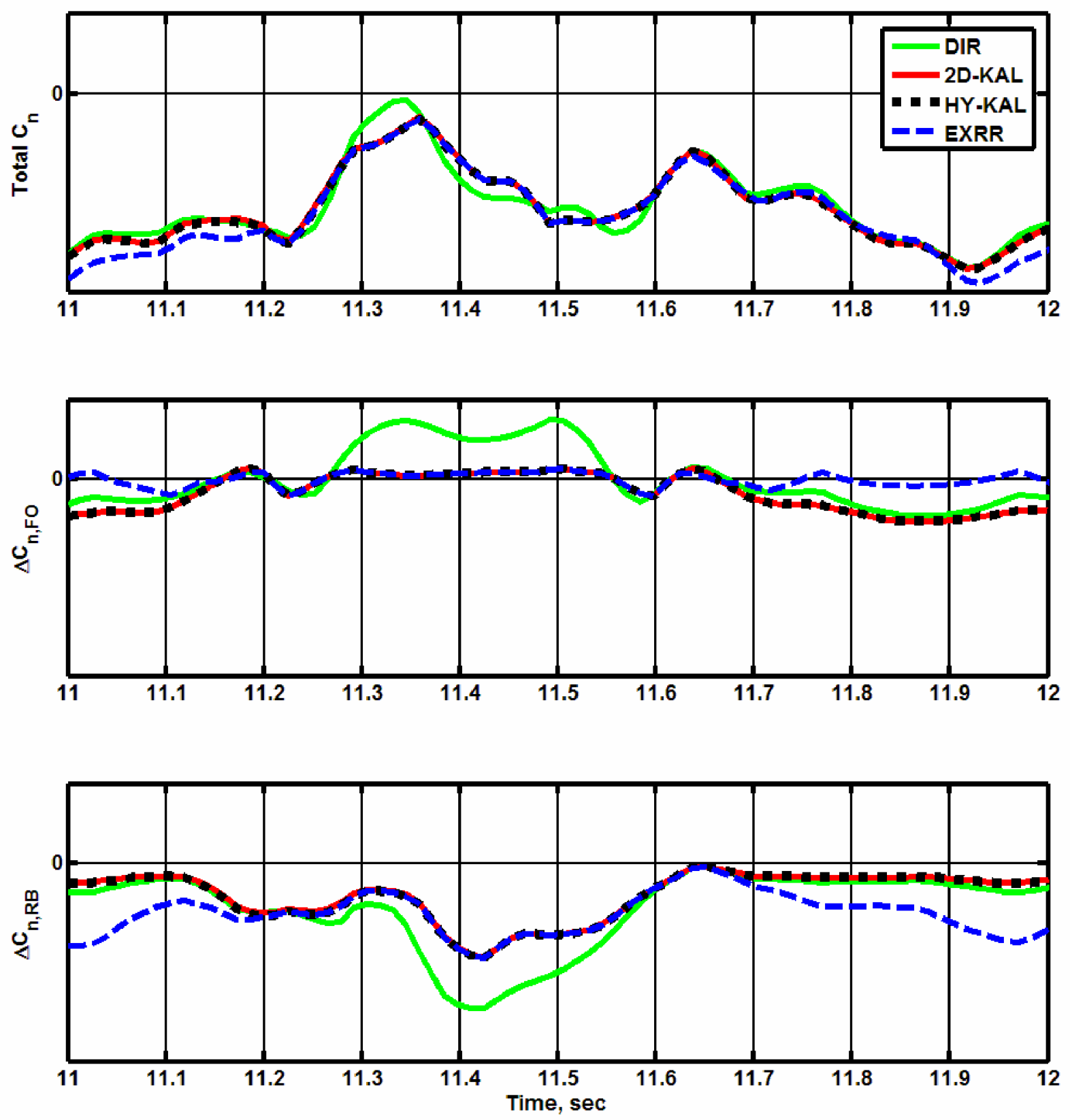

Figure 11. Yawing moment coefficients for a one second time interval of a fully-developed oscillatory spin: Total coefficient, forced oscillation increment, and rotary balance increment. 

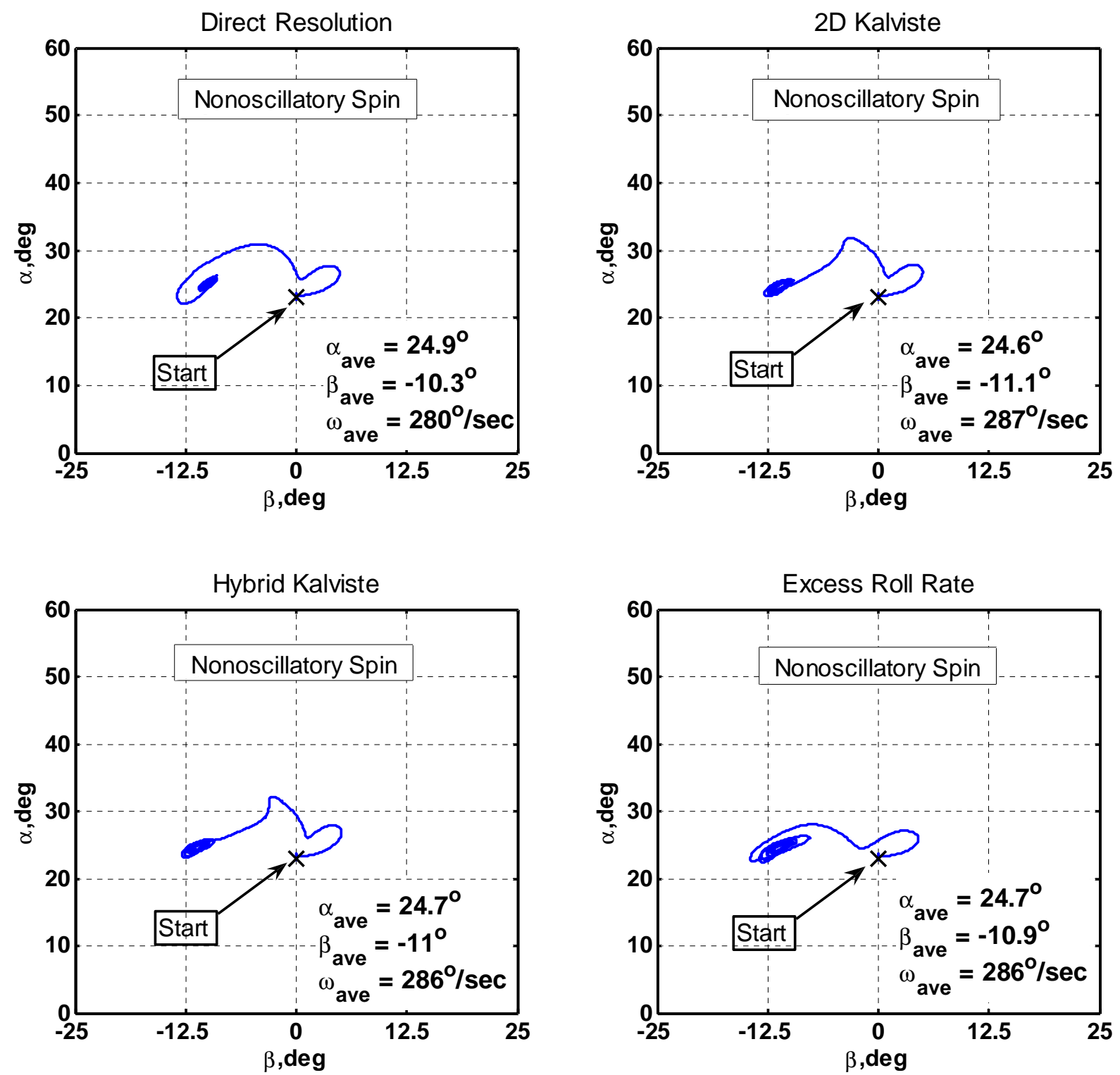

Figure 12. Comparison of blending methods for full pro-spin inputs:

$5.5 \%$ scale, $\mathbf{C G}=25 \% \bar{c}, \delta_{r}=30^{\circ}, \delta_{e}=-30^{\circ}, \delta_{a_{L}}=-20^{\circ}, \delta_{a_{R}}=20^{\circ}$. 

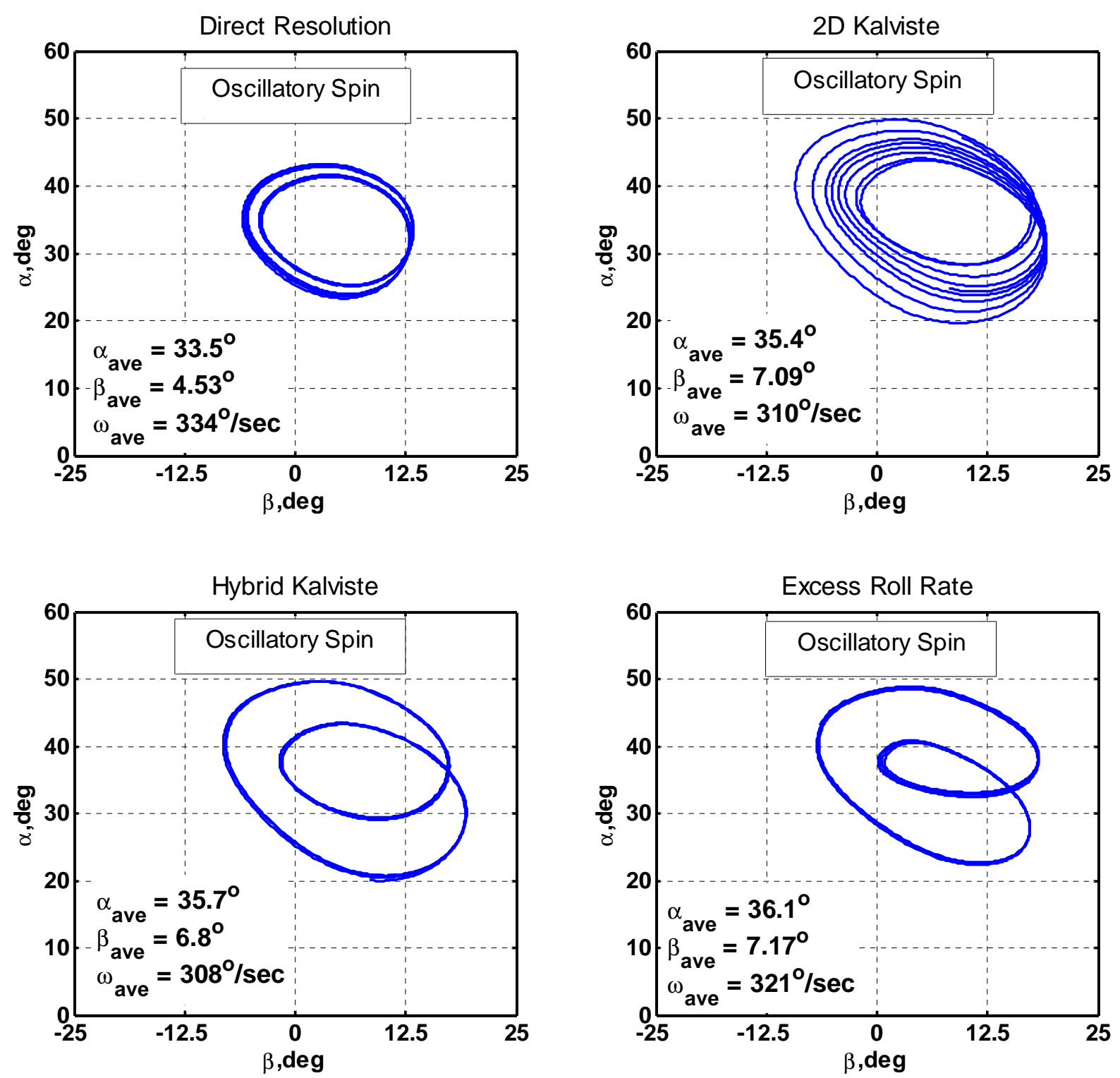

Figure 13. Comparison of blending methods for full pro-spin inputs:

$5.5 \%$ scale, $\mathbf{C G}=25 \% \bar{c}, \delta_{r}=30^{\circ}, \delta_{e}=\delta_{a_{R}}=\delta_{a_{L}}=0^{\circ}$. 

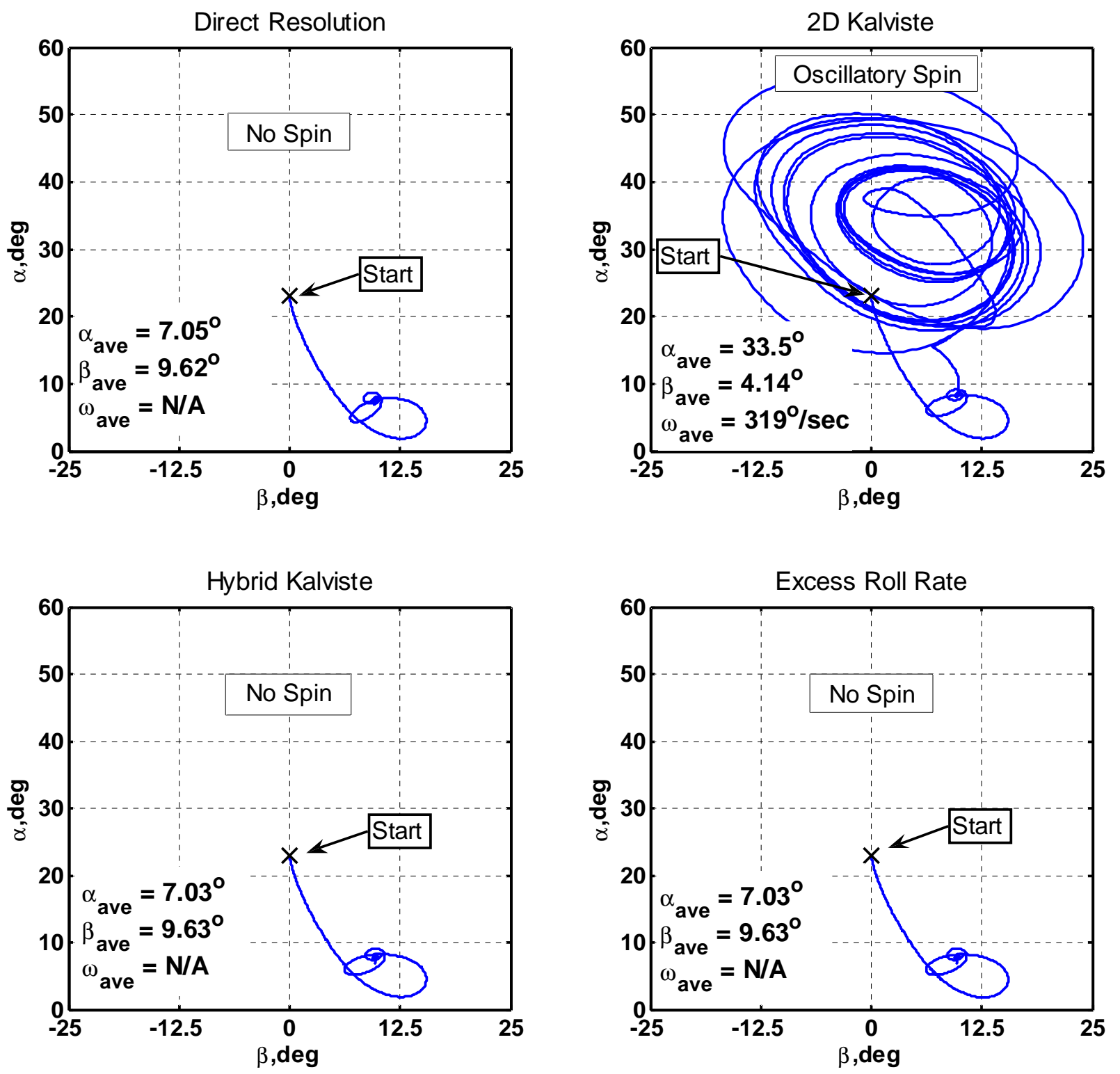

Figure 14. Comparison of blending methods for partial pro-spin inputs:

$5.5 \%$ scale, $\mathbf{C G}=25 \% \bar{c}, \delta_{r}=17^{\circ}, \delta_{e}=\delta_{a_{R}}=\delta_{a_{L}}=0^{\circ}$. 


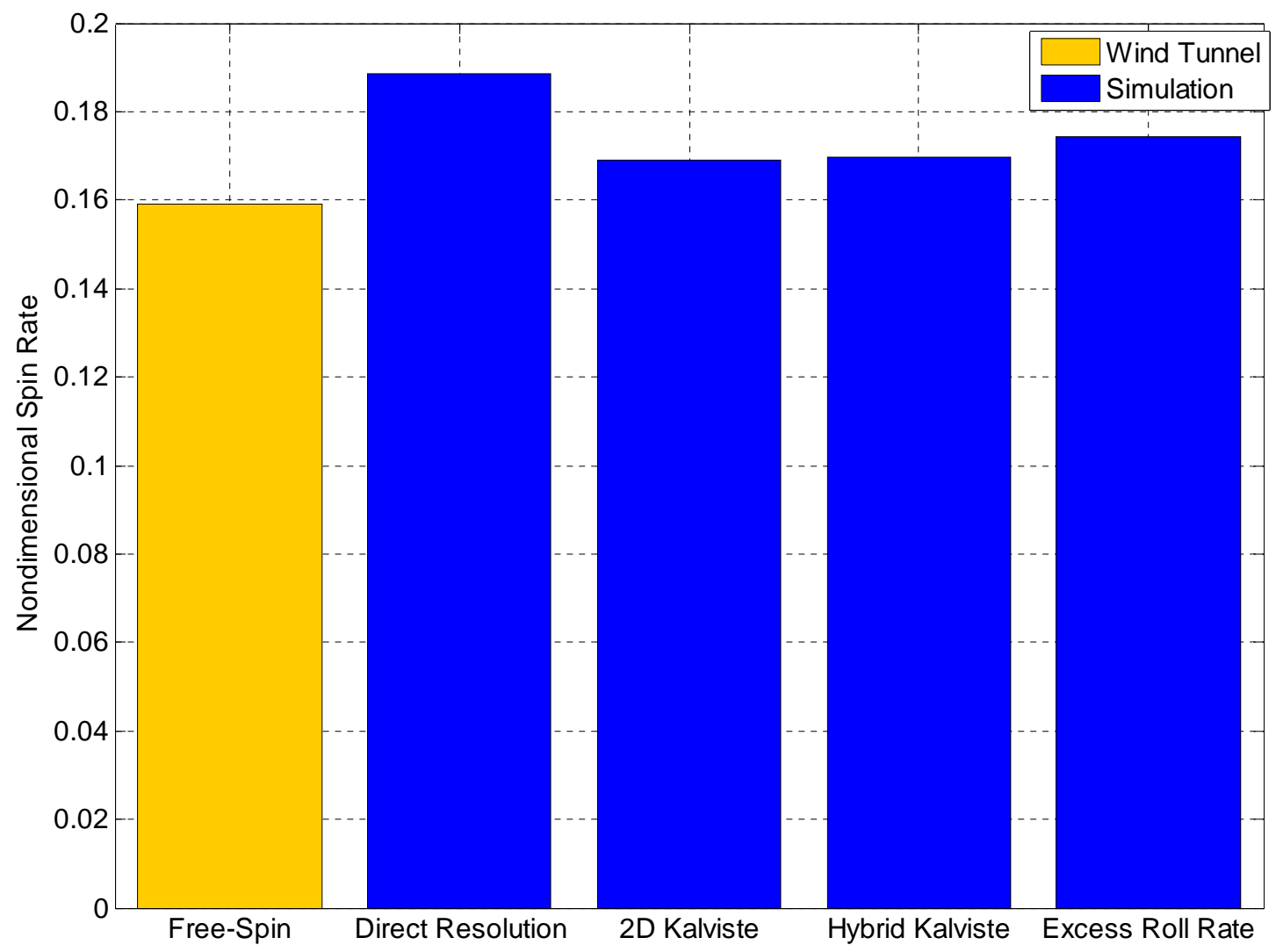

Figure 15. Preliminary free-spin results compared to simulation: average nondimensional spin rate for a fully-developed oscillatory spin, $1 / 49^{\text {th }}$ scale, $\mathbf{C G}=15.2 \% \bar{c}, \delta_{r}=30^{\circ}, \delta_{e}=\delta_{a_{R}}=\delta_{a_{L}}=0^{\circ}$. 